\title{
Research Article \\ The Szlenk Index and the Fixed Point Property under Renorming
}

\section{T. Domínguez Benavides}

Facultad de Matemáticas, Universidad de Sevilla, P.O. Box 1160, Sevilla 41080, Spain

Correspondence should be addressed to T. Domínguez Benavides, tomasd@us.es

Received 25 November 2009; Accepted 19 January 2010

Academic Editor: Tomonari Suzuki

Copyright (c) 2010 T. Domínguez Benavides. This is an open access article distributed under the Creative Commons Attribution License, which permits unrestricted use, distribution, and reproduction in any medium, provided the original work is properly cited.

Assume that $X$ is a Banach space such that its Szlenk index $S_{z}(X)$ is less than or equal to the first infinite ordinal $\omega$. We prove that $X$ can be renormed in such a way that $X$ with the resultant norm satisfies $R(X)<2$, where $R(\cdot)$ is the García-Falset coefficient. This leads us to prove that if $X$ is a Banach space which can be continuously embedded in a Banach space $Y$ with $S_{z}(Y) \leq \omega$, then, $X$ can be renormed to satisfy the w-FPP. This result can be applied to Banach spaces which can be embedded in $C(K)$, where $K$ is a scattered compact topological space such that $K^{(\omega)}=\emptyset$. Furthermore, for a Banach space $(X,\|\cdot\|)$, we consider a distance in the space $D$ of all norms in $X$ which are equivalent to $\|\cdot\|$ (for which $D$ becomes a Baire space). If $S_{z}(X) \leq \omega$, we show that for almost all norms (in the sense of porosity) in $D, X$ satisfies the $\mathrm{w}$-FPP. For general reflexive spaces (independently of the Szlenk index), we prove another strong generic result in the sense of Baire category.

\section{Introduction}

Assume that $(X,\|\cdot\|)$ is a Banach space. The most common aim of the Renorming Theory is to find an equivalent norm which satisfies (or which does not satisfy) certain specific properties. A detailed account of this topic can be found in the monographs [1-3]. This paper focuses on the Renorming Theory in connection with the Fixed Point Theory. It is usually said that a Banach space $X$ satisfies the weak Fixed Point Property (w-FPP) if for every convex weakly compact subset $C$ of $X$, each nonexpansive mapping $T: C \rightarrow C$ has a fixed point. Many geometrical properties of $X$ (uniform convexity, uniform smoothness, uniform convexity in every direction, uniform non-squareness, normal structure, etc.) are known to imply the w-FPP (see, e.g., [4-6] and references therein). However, no characterization of the w-FPP in terms of these properties is known. Therefore, we can regard the w-FPP as an intrinsic property of a Banach space. Since the w-FPP is not preserved under isomorphisms, a very 
natural question in Renorming Theory and Fixed Point Theory would be the following: let $X$ be a Banach space. Is it possible to renorm $X$ so that the resultant space has the w-FPP? This is not generally the case. Indeed, Partington $[7,8]$ has proved that every renorming of $\ell_{\infty}(\Gamma)$ for an uncountable set $\Gamma$ and any renorming of $\ell_{\infty} / c_{0}$ contains an isometric copy of $\ell_{\infty}$ and, consequently, it fails the w-FPP (due to Alspach example [9]). Thus, it would be interesting to identify some classes of Banach spaces which can be renormed to satisfy the w-FPP. For instance, Day et al. [10] have proved that every separable Banach space has a UCED renorming. Since uniform convexity in every direction implies normal structure and this property implies the w-FPP (see, e.g., [4]), we obtain that any separable Banach space can be renormed to satisfy the w-FPP. These arguments do not work for nonseparable spaces because, as mentioned above, there are some Banach spaces which cannot be renormed to satisfy the w-FPP. (In fact, in [10], it is shown that $c_{0}(\Gamma)$ has no UCED renorming if $\Gamma$ is uncountable). Since in [11] an example is given of a reflexive Banach spaces which does not admit any UCED renorming, the following question, which appears in [12, Open Question VI] and [1, Problem VII.3] and which remained unanswered for a long time, seems to be very natural: can any reflexive Banach space be renormed to satisfy the (w)-FPP? In [13] it is shown that this is indeed the case. Actually, the following result is proved in [13]: assume that $X$ is a Banach space such that there exists a bounded one-one linear operator from $X$ into $c_{0}(\Gamma)$. Then, $X$ has an equivalent norm which satisfies the $\mathrm{w}$-FPP. This embedding property is satisfied by a very general class of Banach spaces, for instance subspaces of a space with Markushevich basis, as WCG spaces (and so separable and reflexive spaces), dual of separable spaces as $\ell_{\infty}$, and so forth.

The proof of the result in [13] is strongly based upon some specific properties of the space $c_{0}(\Gamma)$, specially the equality $R\left(c_{0}(\Gamma)\right)=1$, where $R(\cdot)$ is García-Falset's coefficient [14]. It must be noted that any Banach space $Y$ such that $R(Y)<2$ satisfies the $\mathrm{w}$-FPP (see [15]). Thus, it would be natural to extend the above result to any Banach space which can be embedded in more general Banach spaces than $c_{0}(\Gamma)$, but still satisfying $R(Y)<2$. In [16] we prove this extension in the following sense: assume that $Y$ is a Banach space such that $R(Y)<2$, where $R(\cdot)$ is García-Falset's coefficient, and $X$ is a Banach space which can be continuously embedded in $Y$. Then, $X$ can be renormed to satisfy the w-FPP.

In this paper we will use the Szlenk index to show a wide class of Banach spaces $X$ which can be renormed to satisfy $R(X)<2$. The Szlenk index $S_{z}(X)$ [17] is an ordinal number which was introduced to prove that there is no separable reflexive Banach space universal for the class of all separable reflexive Banach spaces. Later, this index has been used in various areas of the geometry of Banach spaces (see [18] for a survey about it). Recently, Raja [19] has proved that if $X$ is an Asplund space and $S_{z}(X) \leq \omega$, then there is an equivalent norm on $X$ such that the dual norm on $X^{*}$ is UKK*. We will show in this paper that this fact leads us to prove $R(X)<2$ when $X$ is endowed with this norm.

On the other hand, if we endow $\Gamma$ with the discrete topology and denote by $K$ the one-point compactification of $\Gamma$, then $c_{0}(\Gamma)$ is isometrically contained in $C(K)$, where $K$ is a topological compact space which satisfies $K^{(2)}=\emptyset$. Thus, if a Banach space can be continuously embedded in $c_{0}(\Gamma)$ then, it can also be embedded in $C(K)$, where $K$ is a scattered compact topological space such that $K^{(\omega)}=\emptyset$. Since $C(K)$ satisfies the w-FPP [20] when $K$ is a scattered compact topological space $K$ such that $K^{(\omega)}=\emptyset$, another natural question would be the following: assume that $X$ is a Banach space which can be continuously embedded in $C(K)$ for some $K$ as above. Can $X$ be renormed to satisfy the $w$-FPP? Using the results about the Szlenk index and the main result in [16], we can prove that this is indeed the case. Nominally, since $S_{z}(C(K)) \leq \omega$ if (and only if) $K$ is as above, we obtain the following: let $C(K)$ be the 
space of real continuous functions defined on a scattered compact topological space $K$ such that $K^{(\omega)}=\emptyset$. Then, it can be renormed in such a way that $R(C(K),\|\cdot\|)<2$ (where $\|\cdot\|$ is the new norm) and the dual norm is UKK*. In order to better understand the relevance of this result, note that in the metrizable case, if $K^{(\omega)}=\emptyset$, then $C(K)$ is isomorphic to $c_{0}$ and, consequently, there exists an equivalent norm $\|\cdot\|$ such that $R(C(K),\|\cdot\|)=1$. From this result and the main result in [16], we can easily deduce that if a Banach space can be continuously embedded in $C(K), K$ as above, then it can be renormed to satisfy the w-FPP. (In [16] the same result for $C(K)$ was obtained by a direct and very technical method). This is a strict improvement of the result in [13], because, as proved in [21], when $K$ is a Ciesielski-Pol's compact, then $K^{(3)}=\emptyset$, but $C(K)$ cannot be continuously embedded in $c_{0}(\Gamma)$ for any set $\Gamma$.

In the last section, for a Banach space $(X,\|\cdot\|)$, we consider a metric in the space $P$ of all norms in $X$ which are equivalent to $\|\cdot\|$, and note that $D$ becomes a Baire space for the corresponding metric topology. If $S_{z}(X) \leq \omega$, we show that for almost all norms (in the sense of porosity) in $D, \mathrm{X}$ satisfies the w-FPP. We finish with another strong generic result in the sense of Baire category for general reflexive spaces (without any assumption on the Szlenk index).

\section{Szlenk Index and Fixed Points}

We start reminding some definition and stating the previous results which we will use.

Definition 2.1. Let $M$ be a topological space and $A$ a subset of $M$. The set $A$ is said to be perfect if it is closed and has no isolated point, that is, $A$ is equal to the set of its own accumulation points. The space $M$ is said to be scattered if it contains no perfect nonvoid subset.

If $A$ is a subset of a topological space $M$, the derived set of $A$ is the set $A^{(1)}$ of all accumulation points of $A$. If $\alpha$ is an ordinal number, we define the $\alpha$ th-derived set by transfinite induction:

$$
A^{(0)}=A, \quad A^{(\alpha+1)}=\left(A^{(\alpha)}\right)^{(1)}, \quad A^{(\lambda)}=\bigcap_{\alpha<\lambda} A^{(\alpha)},
$$

where $\lambda$ is a limit ordinal.

Let us recall the definition of García-Falset's coefficient.

Definition 2.2 (see [14]). Let $X$ be a Banach space. The coefficient $R(X)$ is defined by

$$
R(X)=\sup \left\{\liminf \left\|x_{n}+x\right\|: x_{n} \text { is weakly null with }\left\|x_{n}\right\| \leq 1,\|x\|=1\right\} .
$$

Theorem 2.3 (see [15]). Let $X$ be a Banach space such that $R(X)<2$. Then, $X$ satisfies the $w$-FPP.

Theorem 2.4 (see [16]). Let $Y$ be a Banach space such that $R(Y)<2$. Assume that $X$ is another Banach space, such that there exists a continuous one-to-one mapping $J: X \rightarrow Y$. Then, $X$ can be renormed to satisfy the w-FPP.

Definition 2.5. Let $X$ be a Banach space with dual $X^{*}$. We say that the dual norm is $\mathrm{UKK}^{*}$ if for every $\varepsilon>0$ there is $\theta(\varepsilon)>0$ such that every $u \in B_{X^{*}}$ with $\|u\|>1-\theta(\varepsilon)$ has a weak ${ }^{*}$ open neighborhood $U$ with diam $\left(B_{X^{*}} \cap U\right)<\varepsilon$. 
We remind the definition of the Szlenk index. Following the survey [18], we consider a more general definition than that in [17]. However, both definitions are identical for separable spaces which do not contain $\ell_{1}$.

Definition 2.6. Let $X$ be a Banach space and $X^{*}$ its dual. For any bounded subset $A \subset X^{*}$, we define a Szlenk derivation by $\langle A\rangle_{\varepsilon}^{\prime}=\left\{u \in A\right.$ : for every $\mathrm{w}^{*}$-neighborhood $U$ of $u$, $\operatorname{diam}(A \cap$ $U) \geq \varepsilon\}$. By iteration, the sets $\langle A\rangle_{\varepsilon}^{\gamma}$ are defined for any ordinal number $\gamma$, taking intersection in the case of limit ordinals. The indices $S_{z}(X)_{\varepsilon}$ are ordinal numbers defined as

$$
S_{z}(X)_{\varepsilon}=\inf \left\{\gamma:\left\langle B_{X^{*}}\right\rangle_{\varepsilon}^{\gamma}=\emptyset\right\}
$$

if such an ordinal exists. Otherwise, we write $S_{z}(X)_{\varepsilon}=\infty$. Finally the Szlenk index is defined by $S_{z}(X)=\sup _{\varepsilon>0} S_{z}(X)_{\varepsilon}$.

Remark 2.7. It is known (see [18, Theorem 2] or [1, Theorem 5.2]) that $S_{z}(X) \neq \infty$ if and only if $X$ is an Asplund space. Since our results apply for Banach spaces satisfying $S_{z}(X) \leq \omega$, from now on, we will only consider Asplund spaces.

Theorem 2.8 (see [19]). Let $X$ be an Asplund space with $S_{z}(X) \leq \omega$. Then, there is an equivalent norm on $X$ such that the dual norm on $X^{*}$ is UKK*.

Let $K$ be a compact topological space. It is known (see, e.g., [1, Lemma 8.3]) that $C(K)$ is an Asplund space if and only if $K$ is scattered. For special scattered sets, we have a more precise result.

Theorem 2.9 (see [18, Theorem 24]). Let $K$ be a scattered compact space. The following assertions are equivalent:

(i) $S_{z}(C(K)) \leq \omega$,

(ii) $K^{(\omega)}=\emptyset$. lemma.

We will use the equivalent definition of the $\mathrm{UKK}^{*}$ property given by the following

Lemma 2.10. Assume that $X$ is a Banach space. Then the dual norm is UKK* if and only if for every $\varepsilon>0$, there exists $\delta>0$ such that if $\left\{u_{\alpha}\right\}$ is a net in the unit ball of $X^{*}$ convergent to $u$ in the weak ${ }^{*}$ topology such that $\lim _{\alpha}\left\|u_{\alpha}-u\right\|>\varepsilon$, then $\|u\|<1-\delta$.

Proof. Assume that the above condition is satisfied and let $\varepsilon>0$. Suppose that diam $U \cap B_{X}^{*}>\varepsilon$ for every open neighborhood of $u$ in the weak*-topology. We can choose $u_{U} \in B_{X^{*}} \cap U$ such that $\left\|u_{U}-u\right\|>\varepsilon / 3$. Then, $\left\{u_{U}\right\}$ is a net in $B_{X^{*}}$ convergent to $u$ in the weak*-topology. Taking a subnet $\left\{u_{\alpha}\right\}$ of $\left\{u_{U}\right\}$ such that $\lim _{\alpha}\left\|u_{\alpha}-u\right\|$ exists, we obtain $\|u\| \leq 1-\delta(\varepsilon / 3)$. Conversely, assume that the dual norm is UKK*. Let $\left\{u_{\alpha}\right\}$ be a net in $B_{X^{*}}$ convergent to $u$ in the weak*topology such that $\lim _{\alpha}\left\|u_{\alpha}-u\right\|>\varepsilon$. Let $U$ be an open neighborhood of $u$ in the weak*topology. There exist $\alpha_{0}$ such that for every $\alpha \geq \alpha_{0}$ we have $\left\|u_{\alpha}-u\right\|>\varepsilon$ and $u_{\alpha} \in U$. Thus diam $U \cap B_{X^{*}}>\varepsilon$, which implies $\|u\| \leq 1-\theta(\varepsilon)$.

Remark 2.11. Note that the above notion implies the sequential-UKK ${ }^{*}$ condition, that is, the dual norm is (sequentially)-UKK* if for every $\varepsilon>0$, there exists $\delta>0$ such that if $\left\{u_{n}\right\}$ is a 
sequence in the unit ball of $X^{*}$ convergent to $u$ in the weak* topology such that $\left\|u_{n}-u\right\|>\varepsilon$, then $\|u\|<1-\delta$. Both conditions are equivalent if either $X$ is separable (and, consequently, the weak*-topology restricted to bounded subsets of $X^{*}$ is metrizable) or $X$ is reflexive (due to the angelicity of weak compact sets).

Theorem 2.12. Let $X$ be an Asplund space with $S_{z}(X) \leq \omega$. Then, there is an equivalent norm $|\cdot|$ on $X$ such that $R((X,|\cdot|))<2$ and, hence, $(X,|\cdot|)$ satisfies the $w$-FPP.

Proof. By Theorem 2.8, there exists an equivalent norm on $X$, such that the dual norm satisfies the UKK ${ }^{*}$ property. We follow an argument inspired on that in the proof of Proposition III.11 in [15]. Assume that $\left\{x_{n}\right\}$ is a weakly null sequence in $B_{X}$ and $x \in B_{X}$. For every $n \in \mathbb{N}$, choose $u_{n} \in S_{X^{*}}$ such that $u_{n}\left(x+x_{n}\right)=\left\|x+x_{n}\right\|$. Taking a subsequence, if necessary, we can assume that $\lim _{n}\left\|x+x_{n}\right\|$ does exist. Let $\left\{u_{n_{\alpha}}\right\}$ be a subnet of $\left\{u_{n}\right\}$ which is weak*-convergent to $u$ and such that $\lim _{\alpha}\left\|u_{n_{\alpha}}-u\right\|=\ell$ exists. Assume $\ell \leq 1 / 2$ and choose an arbitrary $\eta>0$. Since $\left\{x_{n_{\alpha}}\right\}$ is a weakly null net, there exists $\alpha_{0}$ such that $\left|u\left(x_{n_{\alpha}}\right)\right|<\eta / 2,\left\|u_{n_{\alpha}}-u\right\|<1 / 2+\eta$ and $\left|u_{n_{\alpha}}(x)-u(x)\right|<\eta / 2$ for every $\alpha \geq \alpha_{0}$. Thus, we have

$$
\begin{aligned}
\left\|x_{n_{\alpha}}+x\right\| & =u_{n_{\alpha}}\left(x_{n_{\alpha}}\right)+u_{n_{\alpha}}(x) \\
& =u(x)+\left(u_{n_{\alpha}}-u\right)\left(x_{n_{\alpha}}\right)+\left(u_{n_{\alpha}}-u\right)(x)+u\left(x_{n_{\alpha}}\right) \\
& \leq\|u\|+\left\|u_{n_{\alpha}}-u\right\|+2 \eta \\
& \leq 1+\frac{1}{2}+2 \eta
\end{aligned}
$$

which implies that $\lim _{n}\left\|x_{n}+x\right\| \leq 3 / 2$. If $\ell>1 / 2$, from Lemma 2.10 we have that $\|u\|<$ $1-\delta(1 / 2)$. Since

$$
\left\|x_{n_{\alpha}}+x\right\|=u_{n_{\alpha}}\left(x_{n_{\alpha}}+x\right) \leq\left|u_{n_{\alpha}}(x)\right|+\left|u_{n_{\alpha}}\left(x_{n_{\alpha}}\right)\right| \leq 1+\left|u_{n_{\alpha}}(x)\right|
$$

we have

$$
\liminf _{n}\left\|x_{n}+x\right\|=\lim _{\alpha}\left\|x_{n_{\alpha}}+x\right\| \leq 1+|u(x)| \leq 1+\|u\| \leq 1+\left(1-\delta\left(\frac{1}{2}\right)\right)<2-\delta\left(\frac{1}{2}\right) .
$$

Thus, $R(X)<\max \{3 / 2,2-\delta(1 / 2)\}$.

Remarks 2.13. (1) Following an argument as in the proof of Proposition III.11 in [15], we can also obtain the condition $R(X)<2$ under the following more general assumption which is usually denoted as $\mathrm{w}_{-}-\mathrm{UKK}^{*}$ property: there exist $\epsilon \in(0,1)$ and $\delta>0$ such that if $\left\{u_{\alpha}\right\}$ is a net in the unit ball of $X^{*}$ convergent to $u$ in the weak*-topology and such that $\lim _{\alpha}\left\|u_{\alpha}-u\right\|>\epsilon$, then $\|u\|<1-\delta$. However, this condition does not yield to an improvement of the above theorem, because if $X^{*}$ satisfies the $\mathrm{w}-\mathrm{UKK}^{*}$ property, there is a renorming of $X$ such that the dual norm satisfies the $\mathrm{UKK}^{*}$ property. Indeed, it is easy to check that the w-UKK* property implies that the Szlenk index $S_{z}(X)_{e}$ is finite for some $\epsilon \in(0,1)$. Since the function $S_{z}(X)_{e}$ is submultiplicative [18, Proposition 4], we have that $S_{z}(X)_{\epsilon^{n}} \leq\left(S_{z}(X)_{\epsilon}\right)^{n}$ and thus $S_{z}(X)_{\epsilon}$ is finite for every positive $\epsilon$. Thus, the existence of an equivalent norm in $X$ such that the dual norm satisfies the UKK* property is a consequence of Theorem 2.8. 
(2) We can also deduce some fixed point properties for the dual norm. First of all, we should mention that if $X$ is an Asplund space, then $X^{*}$ can be continuously embedded in $c_{0}(\Gamma)$ for some set $\Gamma$ [22]. Thus, by the main result in [13], $X^{*}$ has an equivalent (in general nondual) norm which satisfies the w-FPP. On the other hand, we know (see [23, Corollary 5.10]) that property UKK* implies that the coefficient $w * C S\left(X^{*}\right)$ is greater than 1 , where

$$
w * C S\left(X^{*}\right)=\inf \left\{\frac{\lim _{n \neq m}\left\|u_{n}-u_{m}\right\|}{\lim _{n}\left\|u_{n}\right\|}\right\}
$$

and the infimum is taken over all weak*-null sequences $\left\{u_{n}\right\}$ in $X^{*}$ such that both limits exist and $\lim _{n}\left\|u_{n}\right\| \neq 0$. This condition implies that every separable weak*-compact subset of $X^{*}$ has normal structure (see [24, Theorem 2] or [23, Proposition 5.3]). Thus, $X^{*}$ admits a dual equivalent norm such that if $T$ is a nonexpansive mapping defined from a separable weak*compact convex subset $C$ of $X^{*}$ into $C$, then $T$ has a fixed point (see [24, Theorem 1]). If $X$ is reflexive, the separability assumption can be removed, because the condition $W C S\left(X^{*}\right)>$ 1 implies normal structure for weakly compact subsets of $X^{*}$ and we recover the first mentioned renorming result (now, for a dual norm because any equivalent norm is a dual norm in a reflexive space [25]). However, in this case we obtain a stronger result because we have an equivalent norm in $X$ such that $X$ endowed with the new norm satisfies the wFPP and $X^{*}$ endowed with the dual norm satisfies the w-FPP either (Theorem 3.4 in the last section will show a different way to prove a stronger result). Also in the reflexive case, since $X^{*}$ is nearly uniform convex, we can also assure that $X^{*}$ satisfies the $\mathrm{w}$-FPP for nonexpansive multivalued mappings (with compact convex values) (see, e.g., [26]).

Theorem 2.12 jointly with [16, Theorem 2.5] yields to the main result in this paper.

Theorem 2.14. Let $Y$ be a Banach space with $S_{z}(Y) \leq \omega$. Assume that $X$ is another Banach space, such that there exists a continuous one-to-one mapping $J: X \rightarrow Y$. Then, $X$ can be renormed to satisfy the w-FPP.

Assume that $\Gamma$ is an uncountable set. We can consider that $\Gamma$ is endowed with the discrete topology. Let $K$ be the one-point compactification of $\Gamma$. Then, $\left(c_{0}(\Gamma),\|\cdot\|_{\infty}\right)$ is isomorphic to $\left(C(K),\|\cdot\|_{\infty}\right)$ by defining $S: C(K) \rightarrow c_{0}(\Gamma)$ by $S(x(\gamma))=(x(\gamma)-x(\infty))$. Thus any space which can be continuously embedded in $\left(c_{0}(\Gamma),\|\cdot\|_{\infty}\right)$, can be also embedded in $\left(C(K),\|\cdot\|_{\infty}\right)$, where $K^{(2)}=\emptyset$. From Theorems 2.9 and 2.14 , we obtain the following result which strictly improves the main result in [13], because as mentioned in the introduction and proved in [21], there exists a compact set (Ciesielski-Pol's compact), such that $K^{(3)}=\emptyset$, but $C(K)$ cannot be continuously embedded in $c_{0}(\Gamma)$ for any set $\Gamma$. The same result is proved in [16] using a direct but very technical argument.

Corollary 2.15. Let X be a Banach space which can be continuously embedded in $\left(C(K),\|\cdot\|_{\infty}\right)$ for some compact set $K$ such that $K^{(\omega)}=\emptyset$. Then, $X$ can be renormed to satisfy the $w$-FPP.

\section{Genericity of the w-FPP and Szlenk Index}

Following the approach in [27], for a Banach space $(X,\|\cdot\|)$, with closed unit ball $B$, we denote by $D$ the Baire space of all equivalent norms with the metric $\rho(p, q)=\sup \{|p(x)-q(x)|: x \in$ $B\}$. 
In a Baire space, we can regard first category sets as negligible sets. However, we can also consider a deeper notion of negligible set. We should remember that a set $A$ in a topological space $X$ is nowhere dense if its closure has empty interior. If $X$ is a metric space, this fact means that for every $x \in A$ and $r>0$, there exists $y \in X$ and $r^{\prime}>0$ such that $B\left(y, r^{\prime}\right) \subset B(x, r) \backslash A$. A more strict condition is the following.

Definition 3.1. Let $M$ be a metric space. A subset $A$ of $M$ is said to be porous if there exist $0<\beta \leq 1$ and $r_{0}>0$ such that for every $x \in A$ and $0<r \leq r_{0}$, there exists $y \in X$ such that $B(y, \beta r) \subset B(x, r) \cap(M \backslash A)$. A subset $A$ of $M$ is called $\sigma$-porous if $A$ is the union of a countable family of porous sets.

Porous and $\sigma$-porous set can be considered "small" in $M$. In particular a $\sigma$-porous set is obviously of Baire first category and, for $M=\mathbb{R}^{n}$, a $\sigma$-porous set is a null set with respect to the Lebesgue measure.

In [28, Theorem 14], it is proved that if $X$ is a Banach space such that $R(X)<2$, then there exists a $\sigma$-porous set $A \subset D$ such that if $q \in D \backslash A$ the space $(X, q)$ satisfies the w-FPP. From this and Theorem 2.12, we easily obtain the following generic result.

Corollary 3.2. Assume that $X$ is a Banach space with $S_{z}(X) \leq \omega$ and $D$ is the set of all norms in $X$ which are equivalent to the original norm with the metric $\rho(p, q)=\sup \{|p(x)-q(x)|: x \in B\}$. Then, there exists a $\sigma$-porous set $A \subset D$ such that if $q \in D \backslash A$ the space $(X, q)$ satisfies the $w$-FPP.

In particular, we obtain the following generic result, which can be regarded as an improvement of the result in [20] about the w-FPP in $C(K)$.

Corollary 3.3. Assume that $K^{(\omega)}=\emptyset$ and $P$ is the set of all norms in $C(K)$ which are equivalent to the supremum norm with the metric $\rho(p, q)=\sup \{|p(x)-q(x)|: x \in B\}$. Then, there exists a $\sigma$-porous set $\mathbb{A} \subset D$ such that if $q \in D \backslash \mathcal{A}$, the space $(C(K), q)$ satisfies the $w$-FPP.

For general reflexive spaces (independently of the Szlenk index), we can use the main result in [29] to prove a strong generic result in the sense of the Remarks 2.13. If $p$ is a norm in a Banach space $X$, we will denote by $p^{*}$ the dual norm on the dual space $X^{*}$ and by $Q$ the Baire space of all equivalent norms to $\|\cdot\|^{*}$ with the metric $\rho(r, s)=\sup \left\{|r(u)-s(u)|:\|u\|^{*} \leq\right.$ $1\}$.

Theorem 3.4. Let $(X,\|\cdot\|)$ be a reflexive space. There exists a residual subset $R_{0}$ of $D$ (i.e., $D \backslash R_{0}$ is of Baire fist category) such that for every $p \in \mathcal{R}_{0}$, the spaces $(X, p)$ and $\left(X^{*}, p^{*}\right)$ satisfy both the w-FPP.

Proof. By [29, Corollary 2.5], there exist a residual subset $R$ of $D$ and another residual subset $\mathcal{S}$ in $Q$ such that if $p \in \mathcal{R}$ and $s \in \mathcal{S}$, the spaces $(X, p)$ and $\left(X^{*}, s\right)$ satisfy the w-FPP. We claim that the mapping $h: D \rightarrow Q$ defined by $h(p)=p^{*}$ is an homeomorphism from $D$ onto $Q$. Indeed, this mapping is clearly one-one. Moreover, $h$ is onto because any equivalent norm in a reflexive space is a dual norm [25]. It is enough to prove that $h$ is continuous because $h^{-1}$ is similar to $h$. Fixed $p \in D$ and $\epsilon>0$. Denote by $a$ the positive number $\inf \{p(x):\|x\|=1\}$. Assume that $\rho(p, q)<\delta=: \min \left\{\epsilon a^{2} / 4, a / 2\right\}$. Note that $q(x) \geq p(x)-\delta\|x\| \geq a\|x\| / 2$ for every $x \in X$. Furthermore, $p(x) \leq 1$ implies

$$
q\left(\frac{x}{1+\delta\|x\|}\right) \leq 1
$$


and, analogously, $q(x) \leq 1$ implies

$$
p\left(\frac{x}{1+\delta\|x\|}\right) \leq 1
$$

Assume that $\|u\|^{*} \leq 1$ and $q(x) \leq 1$. We have

$$
\begin{aligned}
|u(x)| & \leq\left|u\left(\frac{x}{1+\delta\|x\|}\right)\right|+\left|u\left(x-\frac{x}{1+\delta\|x\|}\right)\right| \\
& \leq \sup \{|u(y)|: p(y) \leq 1\}+\left\|x-\frac{x}{1+\delta\|x\|}\right\| \\
& \leq p^{*}(u)+\delta\|x\|^{2} \leq p^{*}(u)+\varepsilon .
\end{aligned}
$$

Thus $q^{*}(u)<p^{*}(u)+\varepsilon$. Analogously, $p^{*}(u)<q^{*}(u)+\varepsilon$ which implies $\left|p^{*}(u)-q^{*}(u)\right|<\varepsilon$ for every $u$ in the unit ball of $\left(X^{*},\|\cdot\|^{*}\right)$, that is, $\rho\left(p^{*}, q^{*}\right)<\varepsilon$. Finally, defining $\mathcal{R}_{0}=\mathcal{R} \cap h^{-1}(\mathcal{S})$, we conclude the proof.

Remark 3.5. We do not know if a porous version of the above theorem does hold. In fact, we do not know either if Corollary 2.5 in [29] holds in the sense of porosity. Furthermore, the mapping $h: D \rightarrow Q$ defined in the proof of Theorem 3.4 is a homeomorphism, but it is not uniformly continuous. Indeed, the sequence of norms in $\mathbb{R}$, defined by $p_{n}(t)=|t| / n$, is a Cauchy sequence, but the dual sequence $p_{n} *(u)=n|u|$ is not. Thus, the $\sigma$-porosity of $\mathcal{Q} \backslash \mathcal{S}$ does not, in general, imply the $\sigma$-porosity of $p \backslash$ $h^{-1}(\mathcal{S})$.

\section{Acknowledgments}

The author is very grateful to M. Fabian for some valuable comments. The author is partially supported by DGES, Grant BFM2006-13997-C02-01 and Junta de Andalucía, Grant FQM-127. This work is dedicated to W. Takahashi acknowledging his wide and deep legacy in Fixed Point Theory.

\section{References}

[1] R. Deville, G. Godefroy, and V. Zizler, Smoothness and Renormings in Banach Spaces, vol. 64 of Pitman Monographs and Surveys in Pure and Applied Mathematics, Longman Scientific \& Technical, Harlow, UK, 1993.

[2] G. Godefroy, "Renormings of Banach spaces," in Handbook of the Geometry of Banach Spaces, vol. 1, pp. 781-835, North-Holland, Amsterdam, The Netherlands, 2001.

[3] M. Fabian, P. Habala, P. Hájek, V. Montesinos Santalucía, J. Pelant, and V. Zizler, Functional Analysis and Infinite-dimensional Geometry, vol. 8 of CMS Books in Mathematics/Ouvrages de Mathématiques de la SMC, Springer, New York, NY, USA, 2001.

[4] K. Goebel and W. A. Kirk, Topics in Metric Fixed Point Theory, vol. 28 of Cambridge Studies in Advanced Mathematics, Cambridge University Press, Cambridge, UK, 1990.

[5] W. Takahashi, Nonlinear Functional Analysis, Fixed Point Theory and Its Application, Yokohama Publishers, Yokohama, Japan, 2000. 
[6] J. M. Ayerbe Toledano, T. Domínguez Benavides, and G. López Acedo, Measures of Noncompactness in Metric Fixed Point Theory, vol. 99 of Operator Theory: Advances and Applications, Birkhäuser, Basel, Switzerland, 1997.

[7] J. R. Partington, "Equivalent norms on spaces of bounded functions," Israel Journal of Mathematics, vol. 35, no. 3, pp. 205-209, 1980.

[8] J. R. Partington, "Subspaces of certain Banach sequence spaces," The Bulletin of the London Mathematical Society, vol. 13, no. 2, pp. 163-166, 1981.

[9] D. E. Alspach, "A fixed point free nonexpansive map," Proceedings of the American Mathematical Society, vol. 82, no. 3, pp. 423-424, 1981.

[10] M. M. Day, R. C. James, and S. Swaminathan, "Normed linear spaces that are uniformly convex in every direction," Canadian Journal of Mathematics, vol. 23, no. 6, pp. 1051-1059, 1971.

[11] D. N. Kutzarova and S. L. Troyanski, "Reflexive Banach spaces without equivalent norms which are uniformly convex or uniformly differentiable in every direction," Studia Mathematica, vol. 72, no. 1, pp. 91-95, 1982.

[12] W. A. Kirk, "Some questions in metric fixed point theory," in Recent Advances on Metric Fixed Point Theory (Seville, 1995), vol. 48 of Ciencias, pp. 73-97, Universidad de Sevilla, Seville, Spain, 1996.

[13] T. Domínguez Benavides, "A renorming of some nonseparable Banach spaces with the fixed point property," Journal of Mathematical Analysis and Applications, vol. 350, no. 2, pp. 525-530, 2009.

[14] J. García-Falset, "Stability and fixed points for nonexpansive mappings," Houston Journal of Mathematics, vol. 20, no. 3, pp. 495-506, 1994.

[15] J. García Falset, "The fixed point property in Banach spaces with the NUS-property," Journal of Mathematical Analysis and Applications, vol. 215, no. 2, pp. 532-542, 1997.

[16] T. Domínguez Benavides and S. Phothi, "The fixed point property under renorming in some classes of Banach spaces," Nonlinear Analysis: Theory, Methods E Applications, vol. 72, no. 3-4, pp. 1409-1416, 2010.

[17] W. Szlenk, "The non-existence of a separable reflexive Banach space universal for all separable reflexive Banach spaces," Studia Mathematica, vol. 30, pp. 53-61, 1968.

[18] G. Lancien, "A survey on the Szlenk index and some of its applications," RACSAM. Revista de la Real Academia de Ciencias Exactas, Físicas y Naturales. Serie A. Matemáticas, vol. 100, no. 1-2, pp. 209-235, 2006.

[19] M. Raja, "On weak* uniformly Kadec-Klee renormings".

[20] T. Domínguez Benavides and M. A. Japón Pineda, “Fixed points of nonexpansive mappings in spaces of continuous functions," Proceedings of the American Mathematical Society, vol. 133, no. 10, pp. 30373046, 2005.

[21] G. Godefroy, J. Pelant, J. H. M. Whitfield, and V. Zizler, “Banach space properties of Ciesielski-Pol's C (K) space," Proceedings of the American Mathematical Society, vol. 103, no. 4, pp. 1087-1093, 1988.

[22] M. Fabian and G. Godefroy, "The dual of every Asplund space admits a projectional resolution of the identity," Mathematische Annalen, vol. 258, pp. 349-351, 1982.

[23] S. Prus, "Geometrical background of metric fixed point theory," in Handbook of Metric Fixed Point Theory, pp. 93-132, Kluwer Academic Publishers, Dordrecht, The Netherlands, 2001.

[24] T. Domínguez Benavides, J. García Falset, and M. A. Japón Pineda, “The $\tau$-fixed point property for nonexpansive mappings," Abstract and Applied Analysis, vol. 3, no. 3-4, pp. 343-362, 1998.

[25] J. P. Williams, "A "metric" characterization of reflexivity," Proceedings of the American Mathematical Society, vol. 18, pp. 163-165, 1967.

[26] T. Domínguez Benavides and B. Gavira, "The fixed point property for multivalued nonexpansive mappings," Journal of Mathematical Analysis and Applications, vol. 328, no. 2, pp. 1471-1483, 2007.

[27] M. Fabian, L. Zajiček, and V. Zizler, "On residuality of the set of rotunds norms on a Banach space," Studia Mathematica, vol. 91, no. 2, pp. 141-151, 1988.

[28] T. Domínguez Benavides and S. Phothi, Porosity of the Fixed Point Property under Renorming, Fixed Point Theory and Its Applications, Yokohama Publishers, Yokohama, Japan, 2008.

[29] T. Domínguez Benavides and S. Phothi, "Genericity of the fixed point property for reflexive spaces under renormings," to appear in Contemporary Mathematics. 